\title{
Performance Evaluation of Proactive Multipath Routing Protocol for Ubiquitous Networks
}

\author{
Alexandros Ladas \\ WMN Research Group \\ Faculty of Science, \\ Engineering and Computing \\ Kingston University London, UK \\ Email:A.Ladas@kingston.ac.uk \\ Nuwan Weerasinghe \\ WMN Research Group \\ Faculty of Science, \\ Engineering and Computing \\ Kingston University London, UK
}

\author{
Christos Politis \\ WMN Research Group \\ Faculty of Science, \\ Engineering and Computing \\ Kingston University London, UK
}

\begin{abstract}
During the past years, a dramatic increase of peripheral self organised autonomic networks interworking with the traditional infrastructure networks has been highly noticed. Mobile Ad-Hoc Networks (MANETs) have become an interesting topic for research because of their independent nature in terms of network infrastructure and can play a vital role in future Internet communication. A lot of effort has been made in designing efficient routing protocols for MANETs, mainly because of their unique characteristics, such as dynamic topology, high mobility and limited bandwidth. In this paper we evaluate the performance of our Multipath-ChaMeLeon (M-CML) routing protocol and compare to other proactive protocols; Optimised Link State Protocol (OLSR) and DestinationSequenced Distance Vector (DSDV). For this reason, we have considered 2 different scenarios for MANETs by varying the number of nodes and speed values using the NS-3 simulator. The Quality of Service (QoS) metrics we have taken into account are: packet delivery ratio, end-to-end delay, normalised routing load and energy consumption. The acquired results indicate that M-CML routing protocol reduces the effects of unstable communication links, while at the same time improves the performance of the network.
\end{abstract}

Index Terms-ETX, MANETs, Multipath routing, Proactive routing

\section{INTRODUCTION}

Mobile Ad Hoc Networks (MANETs) were introduced to the world by DARPA in the early 1970s, as packet radio networks (PRNET) for military purposes. During the 1980s, DARPA used PRNETs as part of the Survival Radio Network (SURAN) project to a mobile battlefield environment without infrastructure [1]. During the 1990s, the increased interest of the scientific community in this new mobile network technology paradigm led in the creation and establishment of the MANET working group through the Internet Engineering Task Force (IETF) [2].

MANETs can be utilized to establish independent, purpose-built networks, operating in a decentralized manner without relying in any pre-existing infrastructure. Under this light, MANETs are considered as a promising

978-1-5386-3531-5/17/\$31.00 (c) 2017 IEEE solution to address demanding scenarios aiming to provide public protection and disaster relief, especially in cases where traditional networks such as LTE or TETRA are not operating. Their flexible nature in terms of ease of installation, enforces their applicability, ranging from large-scale, mobile, highly dynamic networks, to small, static networks that are constrained by power sources. MANETs can be applied in a variety of situations such as in military sector for day-to-day communications among soldiers, vehicles and headquarters, in commercial sector for emergency communication scenarios (earthquakes, floods, tsunamis).

Despite the broad range of applicability, MANETs are susceptible to a series of challenges. Due to their dynamic nature, nodes within a MANET may enter or leave at any given time, increasing the level of complexity across the network. Furthermore, energy constraints caused by the limited energy capacity of the node's battery, wireless link instabilities produced by node's mobility, as well as the propagation delay negatively impacts MANETs performance. Hence, an optimal routing approach which efficiently exploits the network resources, while enhances the network's QoS performance is the main objective during the design of effective routing algorithms. Within this scope, the distribution of routing messages for the topology identification is one of the main challenging aspects within a MANET, since there is no central routing entity. A lot of efforts have to be dedicated in the design of routing algorithms that take into account a variety of parameters with the view to find the most optimal paths among the nodes.

MANET routing protocols are classified in two main categories named proactive and reactive. Two of the most well-known proactive routing protocols are the Optimized Link State Protocol (OLSR) [3], followed by its successor OLSRv2 [4] and the Destination-Sequenced Distance Vector (DSDV) [5]. The main characteristic of proactive routing, is that the routes are established in advance through the periodic exchange of routing messages, despite the fact that there may not be a 
transmission request. Nodes within a MANET are aware of possible routes by maintaining and updating their routing tables. Proactive routing protocols confine the end-to-end delay enforcing the effective message exchange, but at the same time the constant exchange of routing information, dramatically increases the overhead impacting scalability and energy efficiency.

Contrariwise, reactive protocols such as Ad-hoc Ondemand Distance Vector (AODV) [6] and Dynamic Source Routing (DSR) [7], preserve bandwidth by operating in an on-demand basis. The routes are not preestablished and the routing message exchange mechanism retains in a sleep mode until triggered by a transmission request. Reactive schemes are characterized by the low routing overhead and are considered as energy efficient protocols that can operate well under large network topologies. However, the increased latency due to their on-demand nature, impacts key performance indicators like throughput and delay.

OLSR is a proactive routing protocol commonly used in MANETs. Due to its characteristics it can adapt to dynamic topologies caused by the mobility of the nodes and offers acceptable end-to-end delays during the transmission of data. On the other hand, OLSR employs hop count as the metric which will find the best path for data forwarding. Sometimes, hop count is not the best metric to decide which is the most appropriate route, because it does not take into account the link quality among the nodes. Furthermore, OLSR forwards packets over a single path, which can cause additional delays in cases of link breakages or node failures.

In this paper, we provide a performance evaluation of M-CML [8], a proactive multipath routing protocol based on OLSR. M-CML, in contrast to OLSR, uses Expected Transmission Count (ETX) [9] instead of hop count as metric for obtaining the best path. This feature takes advantage of the periodic exchange of control messages during the link sensing process, as a mechanism to calculate the ETX per link. In addition, M-CML uses multiple path transmission to guarantee message delivery and tackle link instabilities that occur especially in topologies with high mobility.

The paper is organized as follows. The following section gives an overview of M-CML, OLSR, DSDV operation and related work. Section 3 describes in detail the methodology followed to achieve the performance evaluation. Section 4 presents and discusses the simulation results under various Quality of Service (QoS) metrics. Finally, Section 5 concludes the paper and provides considered aims for future work.

\section{THEORETICAL BACKGROUND}

\section{A. Related Work}

Multipath routing can be used to address challenges related to unstable links within the network, high node speeds, and scalability issues [10]. The multipath approach maintains and updates the routing table of participating nodes, while the periodic exchange of control messages, contributes to successfully discovery of all the possible routes without affecting the routing overhead or increasing the energy costs.

Multipath approaches have been adopted by researchers who have implemented a variety of routing approaches. Yi et.al [11] have considered multipath functionality in order to improve TCP data transmission, and according to experiments they managed to improve throughput and reduce the end-to-end delay. Moreover, authors in [12] based their research on OLSR using multipath mechanisms by calculating disjoint and loop free paths. Their results showed that end-to-end delay was improved. The advantage of using ETX as an alternative link metric has influenced researchers to incorporate it in routing protocols. De Couto et al. [9] implemented ETX in DSDV routing protocol and the performance evaluation shows that despite the negative impact of ETX with regards to routing overhead, there is a significant increase in terms of throughput. Liu et al. [13] also made a similar comparison by applying ETX in OLSRv2, showing the improved performance of the protocol only in scenarios that define heavy traffic and high length of the paths. Finally, authors in [14], investigated the performance improvement of DSR protocol, by applying a multipath approach which considers the residual and transmission energy of a potential route. Their method showed enhanced results, compared to DSR, in terms of average residual energy and standard deviation of transmission energy, however no more QoS metrics were provided.

\section{B. OLSR Overview}

OLSR [3] is a table-driven routing protocol, exchanging constant topology information aiming to provide quick route discovery. This core functionality of OLSR is achieved through the periodic distribution of control messages within the participating nodes, which enforces the topology awareness. Hence, data can be transmitted over the most optimal path based on the minimum number of hops required to reach the destination. Another important attribute of OLSR is the use of MultiPoint Relays (MPRs) as part of the control messages operation. The role of MPRs is to eliminate multiple duplicated transmissions by decreasing the improvident emission of broadcast and control messages. OLSRs operation uses 4 different type of control messages as described below:

1) HELLO messages: They are generated and transmitted strictly to 1-hop neighbors. The role of HELLO messages is to perform tasks such as link sensing, neighbor detection and MPR selection and signaling.

2) TC messages: They are generated by the MPRs in order to obtain the topology of the network and store the information in its topology information base, which 
can be utilised to form its routing table. TC messages are forwarded to nodes as mechanism to obtain a global view of the network.

3) Multiple Interface Declaration (MID) and Host and Network Association (HNA) messages: Similar to TC messages, MIDs and HNAs are retransmitted exclusively through the MPRs. MIDs are used to inform the network about the presence of multiple interfaces while HNAs provide connectivity to nodes with non-OLSR interfaces.

OLSR uses the hop count as the default metric for identifying the most optimal route for data packets to be forwarded. Hop count is used to calculate the shortest path based on Dijkstra's algorithm. A great advantage of hop count is that it is easy to be calculated because it does not take into consideration any other factors apart from the hops between the nodes, so it performs good in terms of end to end delay. On the other hand, it does not consider the quality of the links among the nodes which in some cases is very important feature. Furthermore, during OLSRs transmission process, data packets are forwarded over a single path which increases the probability of link and node failures due to circumstances such as high node mobility, unstable links and lossy environments.

\section{DSDV Overview}

The algorithm used by DSDV [5] routing protocol for calculating paths is Bellman-Ford. As OLSR, DSDV is a table-driven protocol which creates and maintains routing tables including all the nodes participating in the network. It also uses hop count as a cost metric to compute the best path from source to destination.The main characteristic of DSDV is its capability of preventing routing loops in the network, by adding a sequence number to all the routing tables. Hence, the participating nodes can distinguish between outdated and new routing information, by taking advantage of the newly added sequence number.

Each node selects independently the routing table's sequence number which increments every time a periodic update takes place. As a prerequisite, the sequence number has to be an even number if a normal update took place. Thus, in the event of a periodic update, all the nodes increment the sequence number by 2 and parse their update to following routing messages. In case of a route is declared as expired, only then the sequence number is incremented by 1 . Each node upon receiving a routing update message, checks the sequence number which has to be higher than the one from previous routing messages. If it is not, the message is discarded. If a node receives an update with same sequence number, then the node keeps only the update with the minimum value of hop count cost metric.

\section{M-CML Overview}

M-CML exploits OLSR's proactive nature as a base and enforces its functionality and performance through the use of an intelligent metric such as the ETX instead of the traditional hop count. ETX is considered as a more reliable solution to address scenarios where the probability of broken links is high, providing reduce proportions of packet loss and increased throughput. Furthermore, M-CML has abolished the single path operation existing in OLSR and has adapted a multipath mechanism, which allows data transmission over multiple routes from the sender to the receiver. The main target of M-CML is to improve the reliability and robustness of the communication of a mobile ad hoc network, by improving QoS metrics such as packet delivery ratio and end-to-end delay. A detailed description of M-CML routing protocol can be found in [8]. The main distinguishing features of M-CML are summarised below:

1) ETX Link Metric: M-CML extends the packet format of HELLO message in order to include ETX values in its tuples. Hence, during the link sensing process of HELLO messages the quality of each link can be calculated and nodes are shortlisted in ascending order in each nodes routing table. Similar pattern is followed for TC messages which are extended to include a vector of ETX values.

2) Multipath Routing: M-CML extends the Route Output process of OLSR by creating a gateway list. The gateway list allocates the routing entry corresponding to the requested destination, parses the already sorted in an ascending order ETX values and transmits the data based on the two minimum ETX values. The reason for selecting 2 paths is that it can offer a desired performance with acceptable complexity [10].

\section{Methodology of Performance Evaluation}

The main objective is to measure the performance of M-CML under different scenarios that have been defined for the purposes of this paper. The behavior of M-CML is compared to OLSR and DSDV routing protocols, in order to study the behavior of the examined protocols in different conditions in terms of node density and various speeds. The performance evaluation is achieved by measuring 4 important QoS metrics; Packet delivery ratio, end-to-end delay, normalised routing load and energy consumption.

\section{A. Simulation Environment}

The performance evaluation of M-CML is performed by carrying out 2 different scenarios. In the first scenario we vary the number of nodes in a simulation area of $1500 \times 1500$ squared meters and $5 \mathrm{~m} / \mathrm{s}$ constant speed. In this scenario the number of sinks is half of the network participants, thus the number of nodes needs to be an even number. The second scenario studies the performance of the routing protocols through different 
speeds in a simulation area of $800 x 800$ squared meters with 16 nodes deployed. We have applied Random Waypoint Mobility Model as a suitable mobility standard that enables nodes to move in random directions within the pre-defined area. Also, Random mobility Allocator is employed, to set up the initial positions of the nodes before the simulation part is triggered. The simulation runs in total for 230 seconds. The first 50 seconds are for warm-up and offer enough amount of time to the nodes to obtain the network topology, while the rest of 180 seconds consist the actual simulation. The main characteristics of the performance evaluation scenarios are presented in Table 1.

TABLE I

Simulation SET

\begin{tabular}{|l||l||l|}
\hline $\begin{array}{l}\text { Network } \\
\text { Parameters }\end{array}$ & Scenario 1 & Scenario 2 \\
\hline Network Topology & $1500 \times 1500 \mathrm{~m}^{2}$ & $800 \times 800 \mathrm{~m}^{2}$ \\
\hline Simulation Time & 230 seconds & 230 seconds \\
\hline Warm-up Time & 50 seconds & 50 seconds \\
\hline Node Speed & $5 \mathrm{~m} / \mathrm{s}$ & $\begin{array}{l}1,5,10,15,20, \\
25 \mathrm{~m} / \mathrm{s}\end{array}$ \\
\hline $\begin{array}{l}\text { Channel } \\
\text { Bandwidth }\end{array}$ & $6 \mathrm{Mbps}$ & $6 \mathrm{Mbps}$ \\
\hline Carrier Frequency & $5 \mathrm{Ghz}$ & $5 \mathrm{Ghz}$ \\
\hline Propagation Model & Friis & Friis \\
\hline MAC Layer & $802.11 \mathrm{a}$ & $802.11 \mathrm{a}$ \\
\hline Number of Nodes & $6,16,26,36,46$ & 16 \\
\hline Source-Sinks Pairs & (No. of nodes)/2 & 8 \\
\hline Initial Energy & 50 Joules & 50 Joules \\
\hline CBR Packet Size & 512 bytes & 512 bytes \\
\hline CBR Data Rate & 4 packets/s & 4 packets/s \\
\hline
\end{tabular}

\section{B. Network and Communication Model Specifications}

Communications among the nodes within a MANET is of paramount importance, hence we are using the ns3 simulator [15] which can represent real scenarios in a trustworthy manner. It has been widely used for research purposes, since it can monitor the behavior of different routing protocols in a variety of environments and analyze their network performance. For the purpose of our simulations we are using Mac802.11a standard (ns3) and modules of the wireless physical layer in order to provide high level of accuracy. The transport protocol which is used is the User Datagram Protocol (UDP) which in contrast to TCP provides a plain transmission model with no need of handshaking processes. The reason of using UDP is that due to MANETs nature, packet loss may occur during transmission process. Finally, Constant Bit Rate (CBR) is employed to generate the network load. The size of the CBR packet is set to 512 bytes, while the number of CBR pairs, (nodes sending and receiving) is set to be equal to the half of the total number of nodes on each case. This is the reason we always have an even number of nodes in our network.

\section{Results}

For the most optimal configuration of M-CML performance, we consider 4 QoS metrics in the MANET domain:

- Packet Delivery Ratio (PDR): The proportion of successful data packets delivered to the destination compared to the total generated data packets,

$$
P=\frac{1}{N} \sum_{i=1}^{N} \frac{R_{i}}{T_{i}}
$$

where $P$ is a fraction of successfully delivered packets, $N$ is the total number of connection flows in the simulation, $i$ is the flow ID, $R_{i}$ is the total number of packets received in flow $i$, and $T_{i}$ is the total number of packets transmitted in flow $i$.

- Average End-to-End Delay (E2ED): The mean time required for the surviving data packet to traverse the distance from the source to the destination,

$$
D=\frac{1}{N} \sum_{i=1}^{N} T_{i}-N_{i}
$$

where $D$ is the average end-to-end delay, $N$ equals to the total number of packets received successfully, $i$ is the packet ID, $T_{i}$ the time when a packet with ID $i$ is received, and $N_{i}$ is the time when a packet with id $i$ is transmitted through a route.

- Normalized Routing Load (NRL): The sum of the transmitted control messages divided by the sum of the delivered data in bytes.

$$
N R L=\sum_{i=1}^{N} \frac{C_{i}}{D_{i}}
$$

where $N$ is the total number of connection flows in the simulation, $C_{i}$ is the number of the transmitted control messages and $D_{i}$ is the received data messages in bytes.

- Energy Consumption (EC): The amount of energy consumed by a node $n_{i}$ during the simulation time is set as $E\left(n_{i}\right)$,

$$
E\left(n_{i}\right)=E_{t x}\left(n_{i}\right)+E_{r x}\left(n_{i}\right)+E_{o}\left(n_{i}\right)
$$

where $E_{t x}\left(\mathrm{n}_{i}\right), E_{r x}\left(\mathrm{n}_{i}\right)$ and $E_{o}\left(\mathrm{n}_{i}\right)$ is the amount of energy node $n_{i}$ wasted for transmitting, receiving and overhearing packets respectively.

The aforementioned evaluation metrics play a significant role in routing protocols evaluation. PDR shows the packet loss of transport protocols, which directly affects the highest value of throughput that can be achieved in the network. The E2ED is also an important factor for MANETs, because high delay values cannot be tolerated. NRL is considered an important metric too, since it affects routing protocols scalability and its efficiency regarding energy consumption. EC is another important aspect which directly affects the nodes operation. 


\section{A. Packet Delivery Ratio}

Figures $1 \& 2$ illustrate the performance of M-CML versus OLSR and DSDV for the 2 considered scenarios. M-CML shows a significant stability in terms of PDR while operating under the different network topologies and nodes speeds. It is also very encouraging the fact that despite OLSR's and DSDV's trend to decrease its proportions of successfully delivered information to the receiver, as the nodes speed increases, M-CML behaves consistently. In Figure 1, the 3 protocols have low PDR when the network topology is comprised of 6 nodes because we have defined a large area with only 6 nodes. For the rest values of node density, M-CML performs significantly better than OLSR and DSDV by delivering $8-10 \%$ and $22 \%-24 \%$ more packets respectively. An identical pattern is followed in Figure 2 where M-CML outperforms OLSR and DSDV in an average of 6\% $10 \%$. This can be attributed to the fact that M-CML exploits its ability to evaluate the quality of the links using an intelligent mechanism such as ETX, while at the same time the multipath routing offers an extra option to the recipient to finally successfully obtain the information.

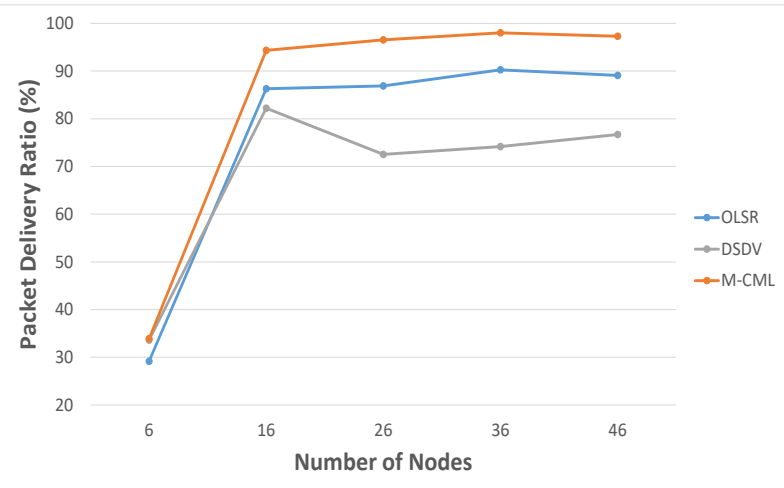

Fig. 1. Packet delivery ratio against various nodes (Scenario 1)

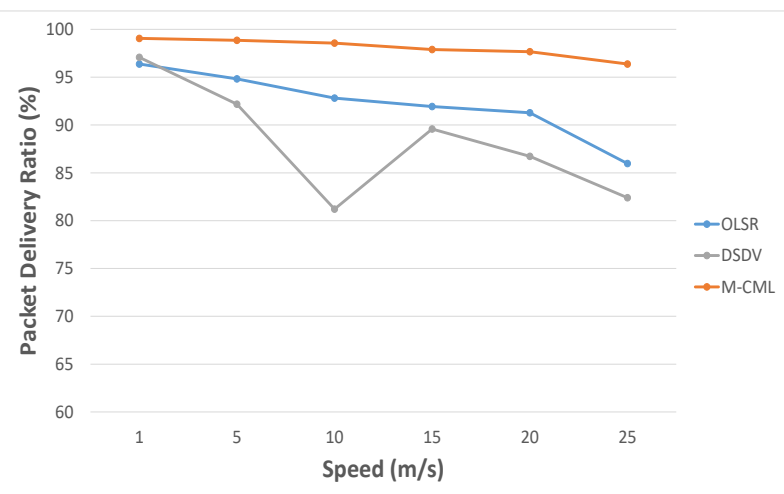

Fig. 2. Packet delivery ratio against various speeds (Scenario 2)

\section{B. End-to-End Delay}

Figures $3 \& 4$ show the behavior of the three protocols in terms of end-to-end delay. It can be observed that the velocity of the nodes as well as operating in various node densities impacts the protocols. In particular, the value of E2ED gradually rises, as the number of nodes and speed respectively increases. However, it shows that MCML operates better compared to OLSR and DSDV in both of the simulations undertaken within the 2 scenarios in this paper. In particular, M-CML manages to transfer the information faster compared to OLSR and DSDV in an average of 3.2 and 6.4 milliseconds respectively for scenario 1 and an average of 0.78 and 1.63 milliseconds respectively for scenario 2 . Despite the fact that M-CML transmits copies of the same message through different paths, increasing the traffic and potential congestion to the receiver, OLSR and DSDV suffer from propagation delay caused by its attribute to forward packets through longer paths based on the traditional hop count, hence it does not take into consideration the quality of the links.

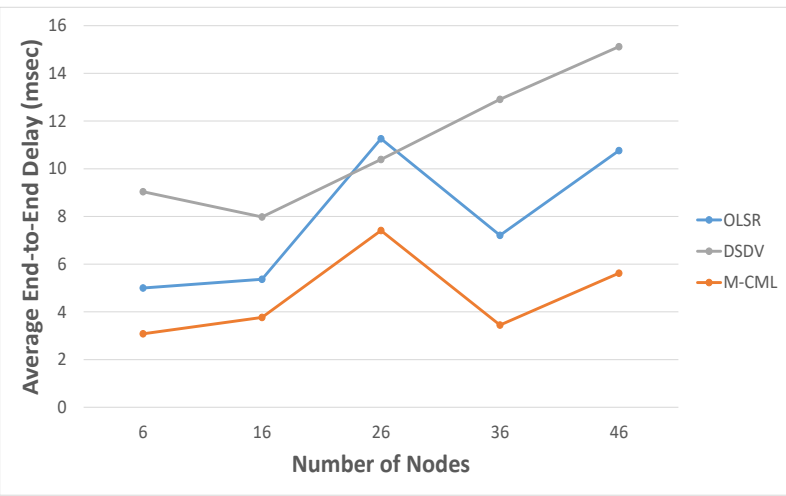

Fig. 3. End-to-end delay against various nodes (Scenario 1)

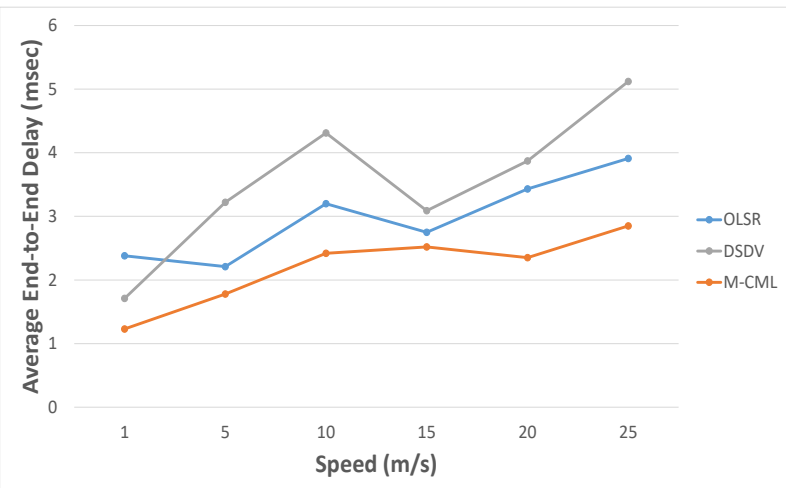

Fig. 4. End-to-end delay against various speeds (Scenario 2)

\section{Normalised Routing Load}

Examining the NRL indicator in Figures $5 \& 6$ we can clearly see that OLSR showed slightly better performance in routing loads compared to M-CML which 
varies on average from 0.02 to 0.03 for the 2 scenarios. This can be charged to the fact that M-CML introduces additional information in the control messages used for the calculation of ETX. This data are incorporated in the HELLO messages which are periodically sent every 2 seconds. Although it impacts the size of the control messages it does not reflect the number of control messages being generated in the examined network topologies. However, M-CML performs clearly better than DSDV in scenario 1 where the node density gets higher generating 0.14 less routing load, and slightly better in scenario 2 generating 0.03 less routing load.

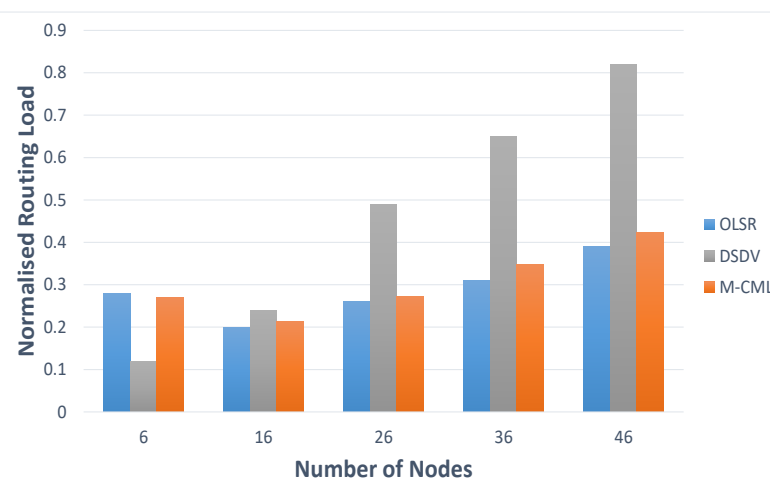

Fig. 5. Normalised routing load against various nodes (Scenario 1)

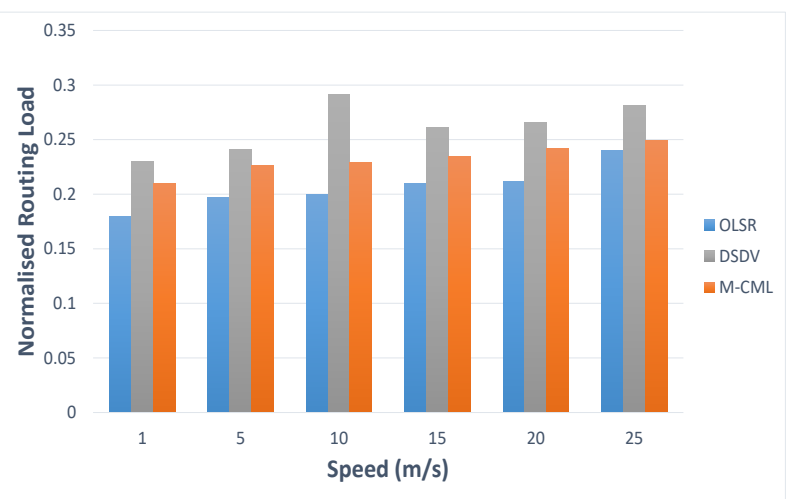

Fig. 6. Normalised routing load against various speeds (Scenario 2)

\section{Nodes Energy Consumption}

Concerning the energy each node consumed in our scenarios OLSR and DSDV show a significantly better performance compared to M-CML. M-CML's attribute to transmit data using the two most optimal paths based on the best ETX value increases the intelligence of the routing approach thereby enhancing some very important QoS parameters such as PDR and E2ED, but on the other hand, it impacts the energy consumption. M-CML's higher energy consumption can be attributed to the fact that it generates improvident data which is distributed throughout the network even when this might not be needed, and also the computation of ETX adds extra computational costs to the network and this impact on the energy consumed.

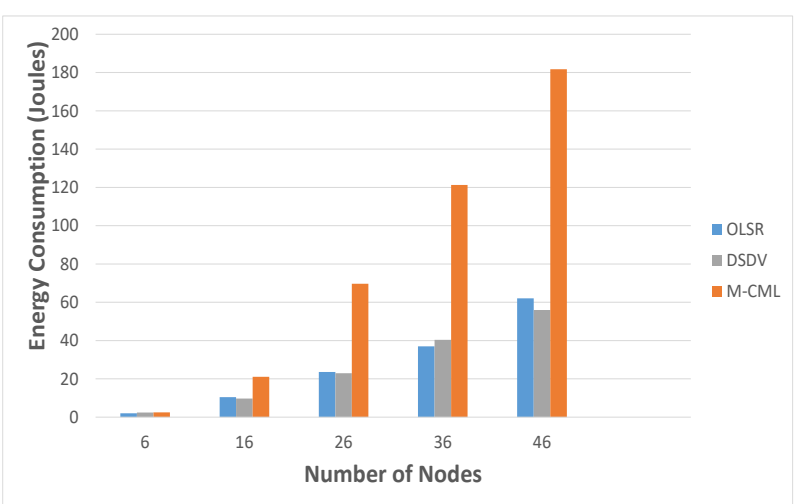

Fig. 7. Nodes energy consumption against various nodes (Scenario 1)

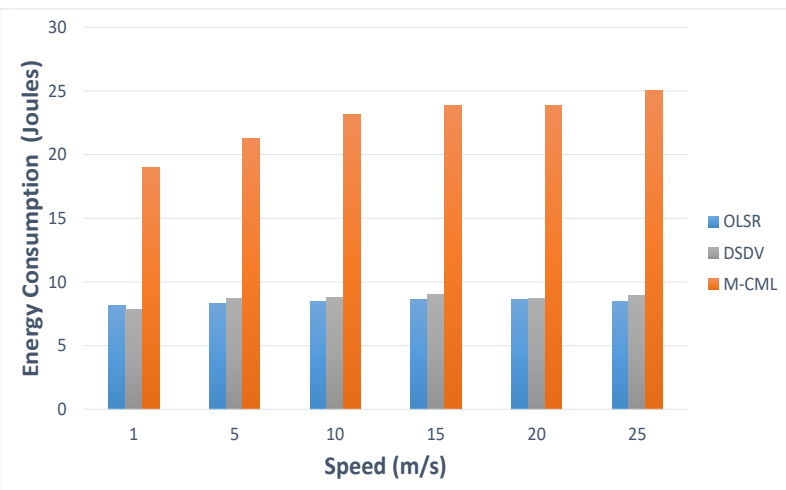

Fig. 8. Nodes energy consumption against various speeds (Scenario 2)

\section{CONClusion AND Future WORK}

Designing routing protocols in MANETs is exciting but also challenging, due to their dynamic nature. In this paper, the performance of M-CML in comparison to OLSR and DSDV has been investigated. For assessing these three protocols, 2 different simulation scenarios were taken into account in order to achieve a detailed evaluation. The performance was measured in a series of QoS metrics: PDR, NRL, E2ED and EC. Having thoroughly analysed the results, we can conclude that there is a trade-off. M-CML routing protocol showed a significant reduction of end-to-end delay and an increased delivery of data packets. However, the energy resources required for the calculation of the ETX combined with the improvident generation and transmission of duplicated packets resulted in increased energy consumptions by the nodes in the network. Using two paths to transmit the same information, the generation of multiple packets could not have been avoided. 
The performance of M-CML can be further enhanced by considering methods to further reduce the emission of duplicated packets, which is the weakest spot of MCML since it directly affects the energy consumption in the nodes and can cause congestion problems in the network. As a matter of future work, we plan to further investigate on the the multipath functionality of M-CML with the view to improve our algorithm in order to dynamically choose between single-path or multipath transmission depending on quality of the links. Moreover, our proposed multipath approach combined with the ETX metric can also be applied as additional module in OLSRv2, which is part of our next steps.

\section{ACKNOWLEDGMENT}

This work was supported by the UK Engineering and Physical Sciences Research Council (EPSRC) Project DARE (Distributed Autonomous and Resilient Emergency Management System) 2017 - 2020 under GCRF (Global Challenges Research Fund) scheme, Grant Reference Number: EP/P028764/1.

\section{REFERENCES}

[1] A. O. Bang and P. L. Ramteke, "Manet: history, challenges and applications," International Journal of Application or Innovation in Engineering \& Management (IJAIEM), vol. 2, no. 9, pp. 249251, 2013.

[2] IETF, "Mobile ad-hoc networks (manet)," The Internet Engineering Task Force.

[3] T. Clausen and P. Jacquet, "Rfc3626: The optimized link state routing protocol," The Internet Engineering Task Force, 2003.

[4] T. Clausen, C. Dearlove, P. Jacquet, and U. Herberg, "The optimized link state routing protocol version 2," Tech. Rep., 2014.

[5] C. E. Perkins and P. Bhagwat, "Highly dynamic destinationsequenced distance-vector routing (dsdv) for mobile computers," SIGCOMM Comput. Commun. Rev., vol. 24, no. 4, pp. 234-244, Oct. 1994.

[6] C. Perkins, E. Belding-Royer, and S. Das, "Ad hoc On-Demand Distance Vector (AODV) Routing," RFC 3561 (Experimental), Internet Engineering Task Force, July 2003.

[7] D. Johnson, Y. Hu, and D. Maltz, "The Dynamic Source Routing Protocol (DSR) for Mobile Ad Hoc Networks for IPv4," RFC 4728 (Experimental), Internet Engineering Task Force, February 2007.

[8] A. Ladas, N. Pavlatos, N. Weerasinghe, and C. Politis, "Multipath routing approach to enhance resiliency and scalability in ad-hoc networks," in 2016 IEEE International Conference on Communications (ICC), May 2016, pp. 1-6.

[9] D. S. J. De Couto, D. Aguayo, J. Bicket, and R. Morris, "A highthroughput path metric for multi-hop wireless routing," Secaucus, NJ, USA, pp. 419-434, Jul. 2005.

[10] J. Yi, A. Adnane, S. David, and B. Parrein, "Multipath optimized link state routing for mobile ad hoc networks," Ad Hoc Netw. vol. 9, no. 1, pp. 28-47, Jan. 2011.

[11] J. Yi, E. Cizeron, S. Hamma, and B. Parrein, "Simulation and performance analysis of $\mathrm{mp}$-olsr for mobile ad hoc networks," in Wireless Communications and Networking Conference, 2008. WCNC 2008. IEEE. IEEE, 2008, pp. 2235-2240.

[12] H. Badis and K. Al Agha, "Qolsr multi-path routing for mobile ad hoc networks based on multiple metrics: bandwidth and delay," in Vehicular Technology Conference, 2004. VTC 2004-Spring. 2004 IEEE 59th, vol. 4, May 2004, pp. 2181-2184 Vol.4.

[13] N. Liu and W. Seah, "Performance evaluation of routing metrics for community wireless mesh networks," in Intelligent Sensors, Sensor Networks and Information Processing (ISSNIP), 2011 Seventh International Conference on, Dec 2011, pp. 556-561.
[14] N. M. Lutimath, L. Suresh, and C. Naikodi, "Efficient source initiated multipath routing protocol for manet," in 2016 International Conference on Signal Processing, Communication, Power and Embedded System (SCOPES), Oct 2016, pp. 1442-1445.

[15] G. F. Riley and T. R. Henderson, "The $n s-3$ network simulator," in Modeling and Tools for Network Simulation, 2010, pp. 15-34. 